\section{OA-021 POINT-OF-NEED DIAGNOSTICS: BIOSURVEILLANCE WITH A DEVICE2CLOUD CAPABILITY IN SIERRA LEONE}

Rashid Ansumana, ${ }^{1}$ C. Taitt, ${ }^{2}$ J.M. Lamin, ${ }^{1}$ K.H. Jacobsen, ${ }^{3}$ S.P. Mulvaney, ${ }^{2}$ T. Leski, ${ }^{2}$ U. Bangura, ${ }^{1}$ D. Stenger ${ }^{2} .{ }^{1}$ MHRL, Njala University, Sierra Leone; ${ }^{2}$ Naval Research Laboratory, United States of America; ${ }^{3}$ George Mason University, United States of America

\subsection{6/bmjgh-2016-000260.28}

Background Infectious diseases contribute to a high burden of diseases globally. Surveillance using low-cost technology, combined with cutting edge platforms offers a path for understanding the disease ecology of locations of interest in resource-poor countries. Our goal was to pilot a bio surveillance system comprising of rapid lateral flow immunoassays, rapid PCR and a cloud database.

Methods The study was carried out in Bo, Sierra Leone at the Mercy Hospital. We recruited 1570 subjects over a period of two years. Inclusion criteria for the study were being febrile, being at least five years of age, living within the city of Bo or its neighbouring villages and agreeing to participate in the study. The assays used included a DPP multiplex lateral flow assay for dengue, Burkholderia pseudomallei, Yersinia pestis, malaria Pf/ Pan, a Film Array PCR platform with multiplex Biothreat and SASFI panels that together detect over 30 pathogen targets. We used a Deki Reader to upload lateral flow images to the cloud database. The Deki reader quantitates test results, such that scores at $\geq 1.75$ are considered positive. A special computer program was designed to upload pdf images of PCR results to the cloud database. The cloud database was designed for automated quality assessment and remote monitoring.

Results Preliminary results show that out of 1570 samples processed by DPP, 30(1.9\%) were positive for Burkholderia, 41 (2.6\%) were positive for Dengue NS1 antigen, 22(1.4\%) were positive for Yesinia pestis fraction 1 antigen, and 340(21.7\%) were positive for malaria. When a cross-section of results obtained by eye was compared with results automatically detected by the D2C platform, there was $95.2 \%$ concordance between results obtained by eye and those obtained automatically by the Deki reader to the cloud database.

Conclusions Active disease surveillance and the ability to remotely monitor activities in peripheral health units are critical needs in many poor countries. Our results provide additional perspectives on the twin problem of surveillance and remote quality assessment. 